\title{
Experimental and Numerical Investigation of the Melting of a Spherical Encapsulated Phase Change Material With Variable Material Data
}

\author{
Valerie Pabst \\ Inst. of Energy and Drive Technology \\ University of Applied Sciences Ulm \\ Ulm, Germany \\ valerie.pabst@thu.de
}

\author{
Robert Güttel \\ Inst. of Chemical Engineering \\ Ulm University \\ Ulm, Germany \\ robert.guettel@uni-ulm.de
}

\author{
Peter Renze \\ Inst. of Energy and Drive Technology \\ University of Applied Sciences Ulm \\ Ulm, Germany \\ peter.renze@thu.de
}

\begin{abstract}
The efficiency of energy applications will gain importance throughout the next years. Development and optimization of future systems, needs to consider power supply, capacity, flexibility of system integrations and economic profitability. Thermal energy storage concepts based on macroencapsulated phase-change material (PCM) promise to fulfill those requirements. One challenge is the change of physical properties of the PCM during its melting, which is here studied numerically and validated by experiments in this contribution.

The numerical model, using the Volume of Fluid method and a detailed description of sensible and latent heat processes, discusses spherical encapsulated PCMs with different melting temperatures. The latent heat source term is computed and corrected through iteration. The numerical results, as verified for a single capsule through an experimental model, can be applied to the research of packed-bed PCMs.
\end{abstract}

Keywords-thermal storage, latent heat, phase change material, numerical simulation, computational fluid dynamics

\section{INTRODUCTION}

Half of Europe's energy demand is for heating and cooling purposes, of which over 60 percent is used for space heating and generation of hot water [1]. To realize an energy revolution, not only the generation of heat is to be improved, but especially its efficiency. Any energy system not having demand and consumption coupled, is in the need of an energy storage. Therefore, it is an important challenge to optimize the thermal energy storages regarding their storage capacity and efficiency. Systems with a higher capacity at the same or smaller storage volume are to be developed in consideration of a flexible system integration, its supply and economic profitability.

Latent heat storages (LHS) promise to meet the demand of higher storage capacities, especially at a small temperature range. Heat is absorbed here through the melting process of the phase change material (PCM), turning the material from solid to liquid. Therefore, heat can be stored without changing the temperature of the system. This latent heat can store ideally up to 5-14 times more energy than the equivalent volume of a sensible heat storage (SHS) material at a small temperature range $[2,3]$. Because of this high thermal storage density several applications of PCMs in thermal energy storages (TES) have been investigated until today. The challenges with PCM investigations are the thermal conductivity of the material, its volume expansion during the melting process, as the density decreases, and therefore the encapsulation of the material. For long term usage a material stability is essential as well. Experimental designs especially of larger LHS are time consuming and thermal processes such as thermal conductivity, viscosity or temperature distributions cannot be monitored closely. Numerical investigations can, however, give insights on these parameters and help design energy efficient storages, before these have to be physically built. Kasibhatla et al. [4] discussed the importance of variable viscosities in numerical PCM models and found a great importance of variable viscosities for accurate melting and settling of a paraffin wax. Vogel and Thess [5] found successful conductivity approximations using the Volume of Fluid (VOF) method with a simplified Boussinesq approximation for temperature and phase fractions, but rather fair results for the modeled velocities. Earlier investigations of Vyshak and Jilani [6] discuss different containers for the PCM to evaluate the melting time. Here, the same mass of PCM is filled in a rectangular, cylindrical and cylindrical shell container, showing the fastest melting rate for the cylindrical shell container. To avoid time-consuming simulations, investigations often neglect the variable material data during the melting process. Dzikevics et al. [7] show in their study a significant better correlation of experiments and numerical model with material data as a function of the temperature.

Different experimental studies of LHS or combined LHS and SHS exist to find improved thermal storage systems [810], but different experimental setups and high efforts are necessary to produce reliable data. Therefore, numerical models are used to reduce experimental efforts for the future combined LHS and SHS application and they are used to be able to improve efficiencies of the storage system beforehand.

Computational fluid dynamics (CFD) simulations allow to analyze the thermal processes more detailed within the encapsulation of the PCM. The investigation of the behavior of the PCM's melting and solidification is of great importance to design efficient thermal storage systems with enhanced capacity. The following paper describes a CFD model with variable material data and a close approximation during the melting temperature range in a 3D model. A simple experiment is conducted to verify the numerical model. 


\section{LATENT HEAT STORAGE}

\section{A. Marcoencapsulated PCM}

Heat transfer into the storage, after the stored time and discharging the storage is a critical aspect. Parameters such as exergy or energy losses, heat transfer surface area and charging periods need to be considered. For latent heat storages, as the phase change is essential in the process, three basic methods to design a LHS are possible

- Exchanging heat at the surface

- Exchanging heat on surfaces within the tank

- Exchanging heat by changing the storage medium

In Fig. 1 these three methods of heat exchanging are described. On the left hand side the heat is transferred over the whole surface, changing the solid PCM to liquid. There is no additional material here. In the middle and right hand figures an additional heat transfer material is needed. In the middle figure heat is transferred from the inside, changing the PCMs face (depicted in red for solid and blue for liquid). This option will be further discussed in the next paragraph. Also possible is a PCM storage where the storage medium is exchanged together with the heat transfer fluid. This is illustrated in the right hand side.
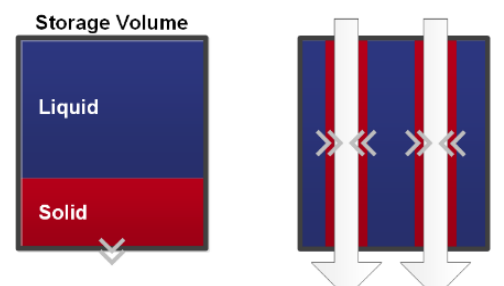

Heat Transfer Fluid

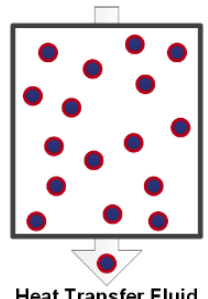

Heat Transfer Fluid
Fig. 1. Options of heat transfer using PCM; cf. [1]

For this application, considering that the heat transfer fluid (HTF) in domestic TES systems is generally water, the heat is exchanged within the tank. As can be seen in Fig. 2, there are also several different options to apply this heat exchanger. Typically known from shell-and-tube heat exchangers the first option has two separate cycles for the HTF and PCM. The second option describes the direct contact method.

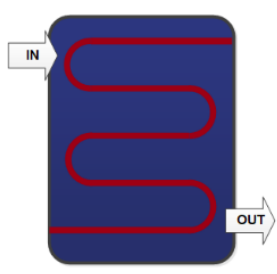

(1)

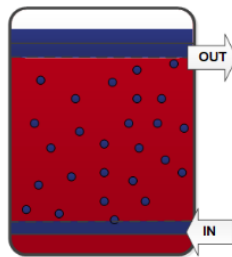

2

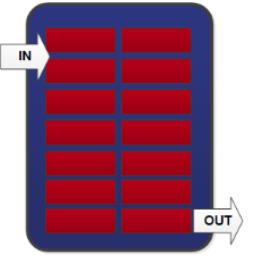

3 PCM

Fig. 2. Heat exchanger types; cf. [1]

Here, the for example metallic structures transfer the heat onto the PCM. The third option is the one to be discussed in this paper. For this method on the right hand side in the illustration, a PCM can be encapsulated in a container and is placed within a tank. This hybrid storage uses the flexibility and high sensible storage capacity of a water storage tank, as

well as the high latent heat of the PCM at the determined temperature.

Previous investigations discussed the shape of the encapsulation container and higher melting rates for spheres than cubes of the same volume. Additionally, spheres offer the smallest possible surface in any shape. This compliments the fact that generally the encapsulation material has the lowest storage capacity of the three materials - HTF, PCM and encapsulation material [11].

\section{B. Common PCM Materials}

Most common PCMs change phase from solid to liquid. However, which PCM is to be chosen for the application depends on several factors, such as: suitable thermal properties as in melting temperature, phase change enthalpy or conductivity, physical properties, especially volumetric expansion and chemical properties as in chemical stability or corrosion. When choosing a PCM it is also important to consider its life cycle for economic reasons. For domestic applications organic and inorganic PCMs are often used, here salthydrates and paraffins are the most common ones. As seen in Fig. 3, PCMs can also be classified as eutectic acids. Paraffins are known for their long life cycles and chemical stability. Salthydrates, though, are cheaper to produce and offer a wider range of phase changes, but they are more reactive towards other materials, hence not as chemically stable.

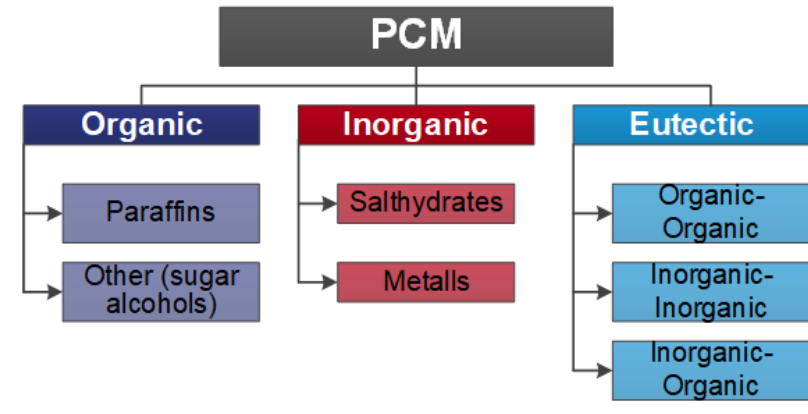

Fig. 3. Commen classification of PCMs; cf. [12]

The investigated PCM is an organic, biodegradable material, but not a paraffin. It offers a high phase change enthalpy and is promised to last over 10,000 cycles without a change in physical properties.

\section{NUMERICAL MODEL}

Transport mechanisms can in detail be modelled with computational fluid dynamics. The Volume of Fluid (VOF) method can be used to determine fraction values $\varphi$ between 0 and 1 , where $\varphi_{s}$ is the solid volume fraction and $\varphi_{1}$ is the liquid volume fraction.

$$
\varphi_{s}+\varphi_{l}=1
$$

This method was first proposed by Hirt et al. [13].The governing Navier-Stokes equations to solve compressible flow are [14] 
Conservation of mass:

$$
\frac{\partial}{\partial t}(\rho)+\nabla \cdot(\rho \boldsymbol{u})=0
$$

Conservation of momentum:

$$
\frac{\partial}{\partial t}(\rho \boldsymbol{u})+\nabla \cdot(\rho \boldsymbol{u} \boldsymbol{u})+\nabla p=\nabla \cdot\left(\mu\left(\nabla \boldsymbol{u}+\nabla \boldsymbol{u}^{T}\right)\right)+\rho \boldsymbol{g}+\boldsymbol{F}_{C S F}
$$

Conservation of energy:

$$
\frac{\partial}{\partial t}(\rho h)+\nabla \cdot(\rho \boldsymbol{u} h)=\nabla \cdot(\lambda \nabla T)+S_{h}
$$

Whereas $\mathbf{u}$ is the velocity, $\mathrm{T}$ is the temperature, $\rho$ represents the density, $\mathrm{p}$ is the pressure, $\mathbf{g}$ is the gravity force causing the sinking of the solid fraction and $t$ is the time. The conservation of energy also accounts for the thermal conductivity $\lambda$ and the total enthalpy $h$. The derivation of the Navier-Stokes equations is discussed in detail in Anderson [15]. Here, $S_{\mathrm{h}}$ is the additional source term representing the latent heat in relation to the volume fraction caused by the phase change. In the momentum equation the change in viscosity is implied by $\mu$. Viscosity, density, thermal conductivity and enthalpy change according to the volume fraction during the phase change. Equation (5) shows the relations for the viscosity, these can be adapted to density, heat transfer coefficient and enthalpy.

$$
\mu=\mu_{s} \varphi_{s}+\mu_{l} \varphi_{l}
$$

In the source term of the momentum equation the continuum surface force between the liquid and solid face is described as $\mathbf{F}_{\mathrm{CSF}}$, with

$$
\boldsymbol{F}_{C S F}=2 \sigma \frac{\varphi_{s} \rho_{s} \kappa_{l} \nabla \varphi_{l}+\varphi_{l} \rho_{l} \kappa_{s} \nabla \varphi_{s}}{\rho_{s}+\rho_{l}}
$$

This includes the surface tension coefficient $\sigma$ as well as the interface curvature $\kappa$ of the surface between solid and liquid state. The curvature is obtained from the operation of the volume fraction relations.

$$
\kappa_{s}=-\kappa_{l}=-\nabla \cdot\left(\frac{\nabla \varphi_{s}}{\left|\nabla \varphi_{s}\right|}\right)
$$

\section{A. Numerical Setup}

The numerical model is implemented in the simulation tool Star CCM+ [16]. To solve both, solid and liquid phases a transient simulation with the VOF model is used.

To include the heat transfer and thermal convection along the boundary of the capsule and PCM, the capsule and its material is modeled as well. A fine grid is necessary to solve transport mechanisms accurately. The grid is fixed, but with layer refinement along the boundary and has approximately 1.07 million cells, including PCM and shell. A polyhedral mesh was chosen.

The segregated solvers of the multiphase temperature and volume flux based flow, as well as the VOF are discretized as $2^{\text {nd }}$ order.

\section{B. Mushy Zone Models}

In order to model melting and settling of the solid phase, formulations are needed to describe the temperature depending relation. As realistic PCMs usually melt within a temperature range, the model also needs to be able to reflect this mushy zone over changing temperature.

The mushy zone represents the area where the solid surface begins to melt and cast into the melted material. This means that $\varphi_{\mathrm{s}}<1$ and $\varphi_{1}<1$. To generate a movement in the melting and solidification process the Carman-Kozeny Mushy Zone Permeability method is implemented. The flow resistance for cells above a solid volume fraction (SVF) of zero is activated, causing a high flow resistance of $10^{6} \mathrm{~kg} / \mathrm{m}^{3} \mathrm{~S}$ to imitate a solid body when the SVF of the PCM is one. For SVF between one and zero, in the mushy zone, the flow resistance is modeled similar to an isotropic porous medium, by using the Carman-Kozney equation to calculate the permeability $[16,17]$ :

$$
K=\frac{\left(1-\varphi_{s}\right)^{3}}{\varphi_{s}^{2} F_{K} c_{s}}
$$

Where $\mathrm{K}$ is the permeability, $\mathrm{c}_{\mathrm{s}}$ is the shape factor and FK is the switching function, implementing the Metzner Slurry Viscosity for small SVF $<0.27$. This Metzner Slurry Viscosity model [18] expresses viscosities as a function of its SVF $\varphi_{s}$

$$
\mu=\mu_{l}\left[1-\left(\frac{\varphi \cdot F_{\mu}\left(\varphi_{s}\right)}{A}\right)\right]
$$

Where $\mu$ is the effective viscosity and $\mu_{1}$ its liquid share. $F_{\mu}$ represents the switching function for the Metzner Slurry Viscosity model and A is a crystal constant depending on the aspect ratio of the surface roughness. The switching functions $F_{K}$ and $F_{\mu}$ are inverse functions that take the critical SVF into account. The here used switching function was proposed by Chan and Stefanesu [19]. As mentioned the change of functions here is defined at $\varphi_{c r}=0.27$, including also the switching function constant $\mathrm{s}$

$$
F_{K}\left(\varphi_{s}\right)=1-F_{\mu}\left(\varphi_{s}\right)=0.5+\frac{\arctan \left[s\left(\varphi_{s}-\varphi_{c r}\right)\right]}{\pi}
$$

For the contribution to the momentum equation is the viscous resistance tensor $\mathrm{P}_{\mathrm{V}}$ that is described by Darcy's term in this simulation setup

$$
P_{V, \text { mushy zone }}=\frac{\mu}{K}
$$

\section{Functions Modelling Material Data}

Next to the numerical set up, the material data during the melting process is described by an iterative approach, depicting the material properties during the phase change as a linear function of the temperature T. This includes density $\rho$, specific heat $c_{p}$ and the thermal conductivity $\lambda$. As can be seen in the following figure, the linear approximation throughout the melting process gives a more accurate insight on the material properties, as opposed to the often used isothermal approximation. 

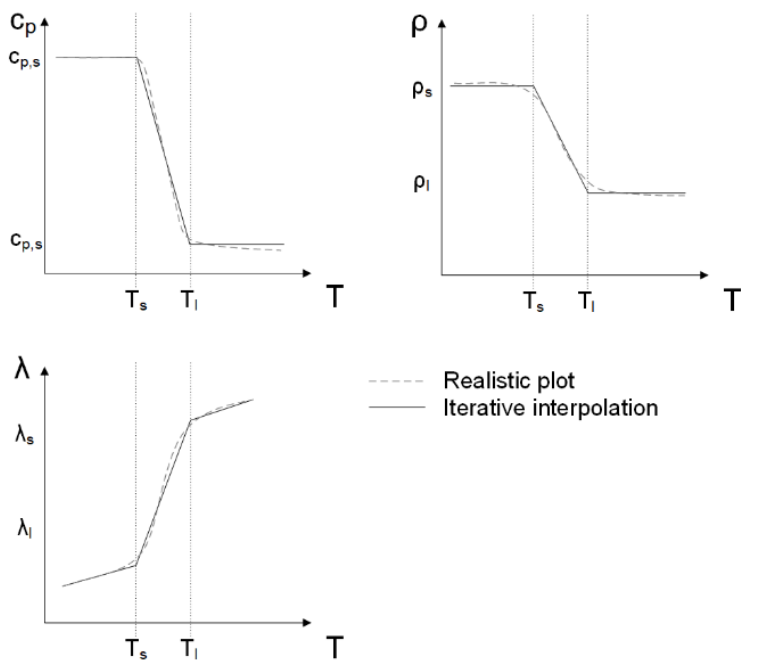

Fig. 4. Iterative interpolation of material properties, showing a linear approximation of variable material properties. Funtions are shown over the change in temperature.

The implemented physical properties are based on the data provided by the producer Croda GmbH. Table I gives an overview of the most important material data used, such as the latent heat, specific heat or thermal conductivity and the melting range.

The PCMs used, expand their volume while changing phase. For Croda53 this accounts to $9 \%$ of the volume.

TABLE I. MATERIAL PROPERTIES OF THE PCM WITH A PHASECHANGE TEMPERATURE OF $53^{\circ} \mathrm{C}$

\begin{tabular}{|l|l|l|l|}
\hline \multirow{2}{*}{ Material properties } & \multirow{2}{*}{ Units } & \multicolumn{2}{|c|}{ CrodaTherm 53 } \\
\cline { 3 - 4 } Density & $\mathrm{kg} / \mathrm{m}^{3}$ & 904 & \multicolumn{1}{|c|}{ Liquid } \\
\hline Viscosity & $\mathrm{Pa} \mathrm{s}$ & Model & 0.05 \\
\hline Specific heat capacity & $\mathrm{J} / \mathrm{kgK}$ & 1900 & 2200 \\
\hline Latent heat & $\mathrm{J} / \mathrm{kg}$ & 0 & 225 \\
\hline Thermal conductivity & $\mathrm{W} / \mathrm{mK}$ & 0.28 & 0.16 \\
\hline Melting temperature & $\mathrm{K}$ & 324.15 & 326.15 \\
\hline
\end{tabular}

TABLE II. BOUNDARY CONDITIONS

\begin{tabular}{|l|l|l|l|}
\hline \multirow{2}{*}{ Physical property } & \multirow{2}{*}{ Units } & \multicolumn{2}{|c|}{ CrodaTherm 53 } \\
\cline { 3 - 4 } & & Initial & Boundary \\
\hline Temperature & K & 315.15 & 331.15 \\
\hline
\end{tabular}

For the boundary conditions a temperature range of $\mathrm{T}=5 \mathrm{~K}$ before and after the melting temperature range is applied. These are the same conditions as conducted in the experimental set up. The measured sphere has an inner diameter of $d_{i}=3 \mathrm{~cm}$ and an outer diameter of $d_{o}=3.2 \mathrm{~cm}$, creating a wall thickness of $0.1 \mathrm{~cm}$. The gravity of the numerical setup is directed into the negative y-axis.

\section{EXPERIMENTAL SETUP}

The experimental setup consists of a thermal bath, adjustable to any temperature in the range of 0 and $200{ }^{\circ} \mathrm{C}$. As it is operated with water and at ambient pressure, temperatures cannot exceed the evaporation point. Temperature sensors within the water cycle and the PCM track its data per second. As sensors are of Type $\mathrm{K}, \varnothing 1 \mathrm{~mm}$, and therefore thin compared to the probe, the heat transfer through the sensor is neglected. The sphere capsule is $3 \mathrm{D}$-printed with a translucent material.

Perforated plates encourage an even and laminar flow around the PCM probe. As can be seen in Fig. 5, supporting ribs keep the sphere filled with PCM in the middle of the measuring section. For each experiment conducted, the temperature at the inlet of the measuring section is set equal to the boundary temperature of the simulation. The mass flow rate is measured with a magnetic-inductive flowmeter (MIF), showing a flow rate of $\dot{\mathrm{V}}=0.745 \mathrm{~m}^{3} / \mathrm{h}$.

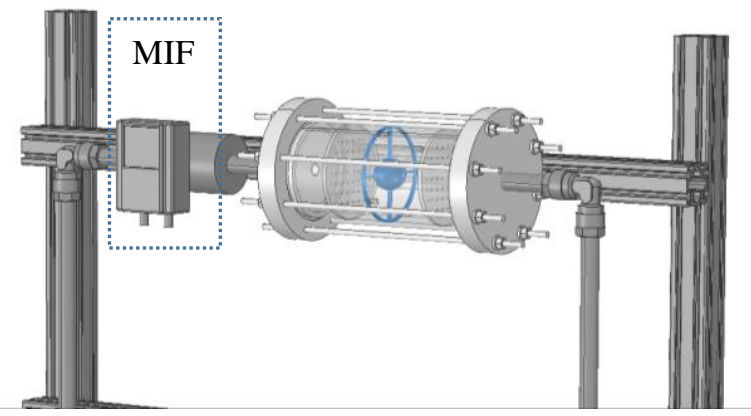

Fig. 5. Visualization of the experimental setup showing the PCM capsule between the perforated plates and position of the MIF.

The probe measured has an inner diameter of $\mathrm{d}_{\mathrm{i}}=3 \mathrm{~cm}$ and a wall thickness of $1 \mathrm{~mm}$, leading to an outer diameter of $\mathrm{d}_{\mathrm{o}}=3.2 \mathrm{~cm}$. The measuring section consists of a $30 \mathrm{~cm}$ long transparent pipe including perforated plates. They are placed in a $7 \mathrm{~cm}$ distance from the inlet and outlet. The probe is fixed in the middle of the measuring section. As can be seen in Table I, the melting range of the PCM is from $\mathrm{T}_{\mathrm{s}}=324.15 \mathrm{~K}$ to $\mathrm{T}_{1}=326.15 \mathrm{~K}$. The inlet temperatures are set according to the simulation and at $\mathrm{T}=5 \mathrm{~K}$ above the maximum melting temperature, as seen in Table II.

\section{RESULTS AND DISCUSSION}

The melting front, the mushy zone and temperature can be observed in the simulation as well as the experimental result described in the next section. Additionally the velocities within the sphere were monitored to get more detailed insights of the melting rate and its causes.

\section{A. Simulation Results}

The Mushy Zone Permeability model shows the modeled viscosities along the melting front and can be compared to the SVF $\varphi$ equivalent time step. Fig. 6 shows this viscous resistance (bottom right sphere) at a time step of $\mathrm{t}=500 \mathrm{~s}$. 

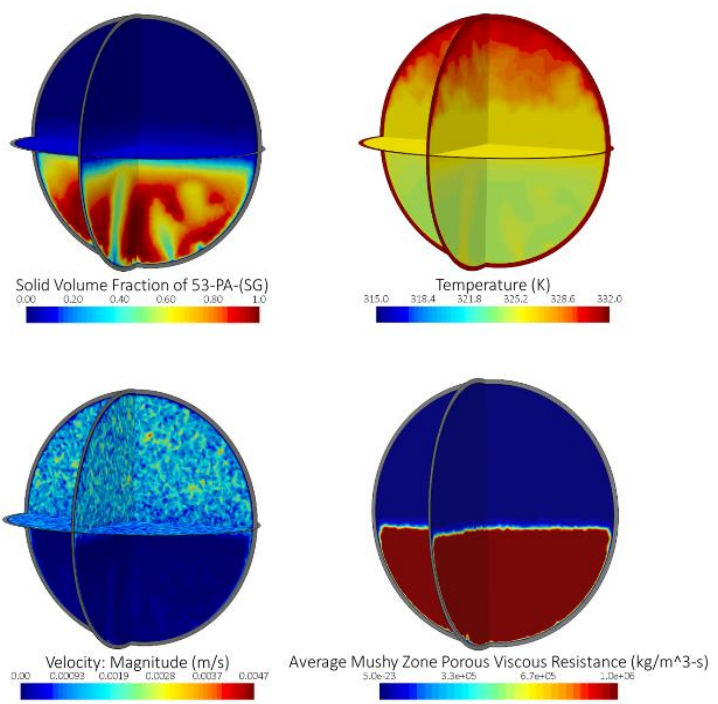

Fig. 6. Visualization of the Mushy Zone Permeability method with its flow resistance at $\mathrm{t}=500 \mathrm{~s}$ compared to the $\operatorname{SVF} \varphi$, velocity $\mathbf{u}$ and termperature $T$ of the PCM

For the SVF, upper left side in Fig. 6, dark red areas show $\varphi=1$ degrading towards dark blue with $\varphi=0$. This gives $\varphi \approx 0.27$, where the switching function is active at the turquois to green range. Here the SVF shows the same border as the Mushy Zone Porous Viscous Resistance in the bottom right picture. For values above $\varphi>0.27$ the Mushy Zone Permeability model sets a high temperature, gradually decreasing the resistance towards the melting front. The path of further melted material close to axis of the sphere can hardly be seen, as it is not yet melted below $\varphi<0.27$.

As it can be seen in the visualization of the SVF as a thin blue line, a thin melted layer develops along the border to the wall. This layers stays thin until the last moments of melting, due to the gravity forcing a movement towards the negative $y$ direction. This velocity and gravity causes the PCM to slide in negative $y$-direction and melt faster. The upper right visualization shows the temperature $T$. As can be seen during the melting process the temperature changes only in the range of $2 \mathrm{~K}$ as the melting range is defined. Red colors along the upper wall of the sphere indicate a rising temperature where the PCM is already fully liquid. As the Mushy Zone Permeability model sets a high flow resistance to particles with $\varphi>0.27$, the velocities for these areas are $\mathbf{u}=0 \mathrm{~m} / \mathrm{s}$. This can be seen in the bottom left picture of Fig. 6. It also shows the movements along the boundary wall in light blue, causing the melted, liquid material to move in the positive y-direction.

Over the simulated time of $t=1000 \mathrm{~s}$ the SVF and temperature were monitored along five lines parallel to the $\mathrm{x}$ axis with ten measuring points each. Each line has a distance of $5 \mathrm{~mm}$ to the next measuring line probe. In Fig. 7 the SVF of five of the measuring points is displayed over time. SVF 1 (dark blue, full line) accounts for the measuring point with the highest y-value decreasing up to SVF 5 (blue, dashed-dotted line) with the lowest y- value. SVF 2 is displayed in red and dotted, SVF 3 in grey and short dashes and SVF 4 in blue with long dashes.
This shows the quick melting of the measurement with the highest y-value, here SVF 1. It can be seen that all curves approach zero more gradually starting from a y-value below $\varphi=0.27$. This indicates the used switching function and the change from the Mushy Zone Permeability Model to the Metzner Slurry Viscosity Model. As SVF 5 is a measuring point $5 \mathrm{~mm}$ above the bottom wall of the sphere, the whole melting process is finished shortly after this line reaches zero. The overall melting process lasts $\mathrm{t}=980 \mathrm{~s}$.

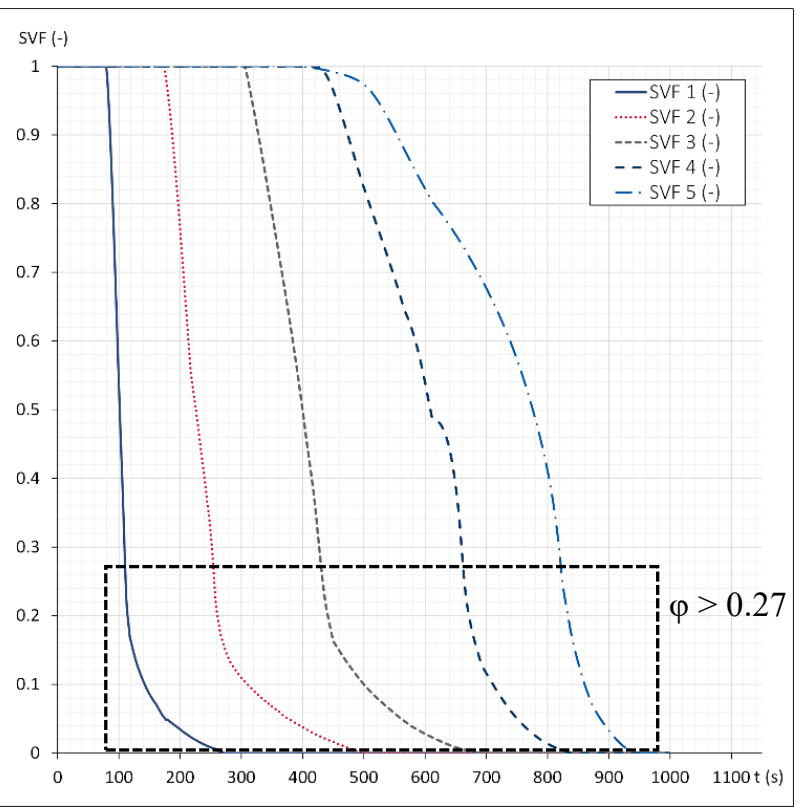

Fig. 7. Different measuring points of solid volume fractions each $5 \mathrm{~mm}$ apart from each other; starting with SVF 1 (-) $5 \mathrm{~mm}$ in negative y-direction from the top of the sphere and continuing every $5 \mathrm{~mm}$.

\section{B. Comparison of Simulation and Experiment}

The monitored temperature $\mathrm{T}$ of the simulation is compared to the temperature data from the experiment in Fig. 8.

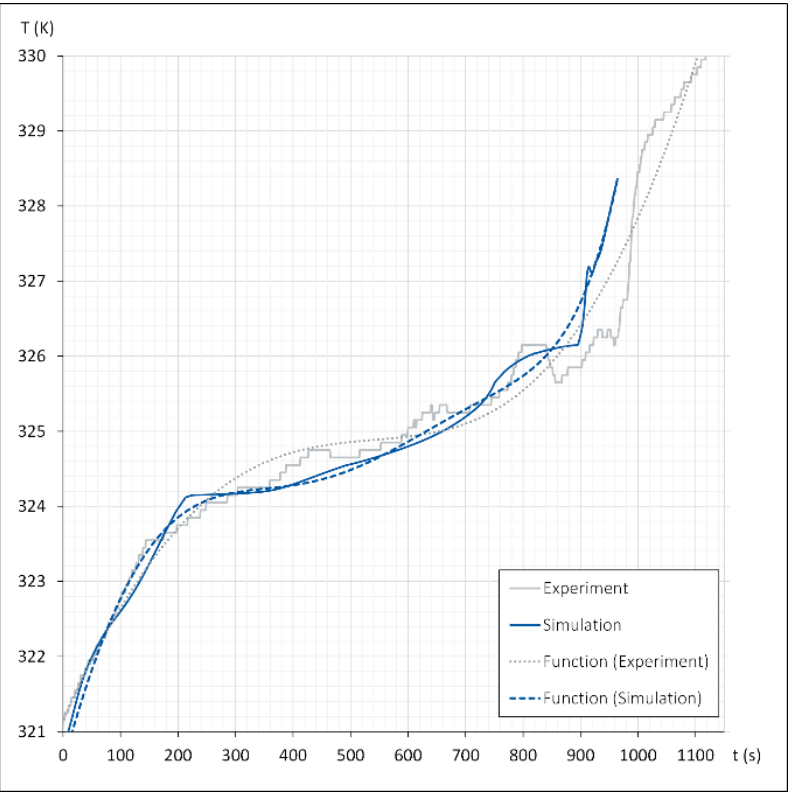

Fig. 8. Temperatures curves of the simulation (blue) and experiment (grey) over time. Functions to compensate small deviations are displayed dotted. 

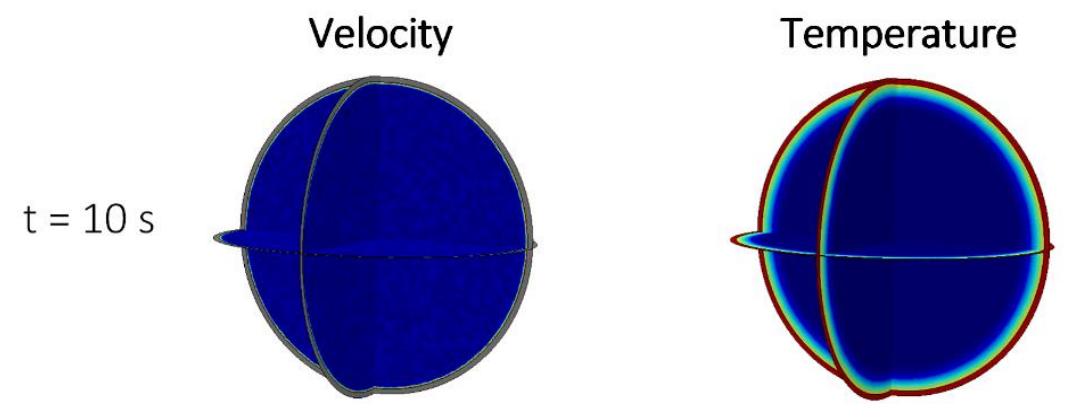

\section{Solid Volume Fraction}
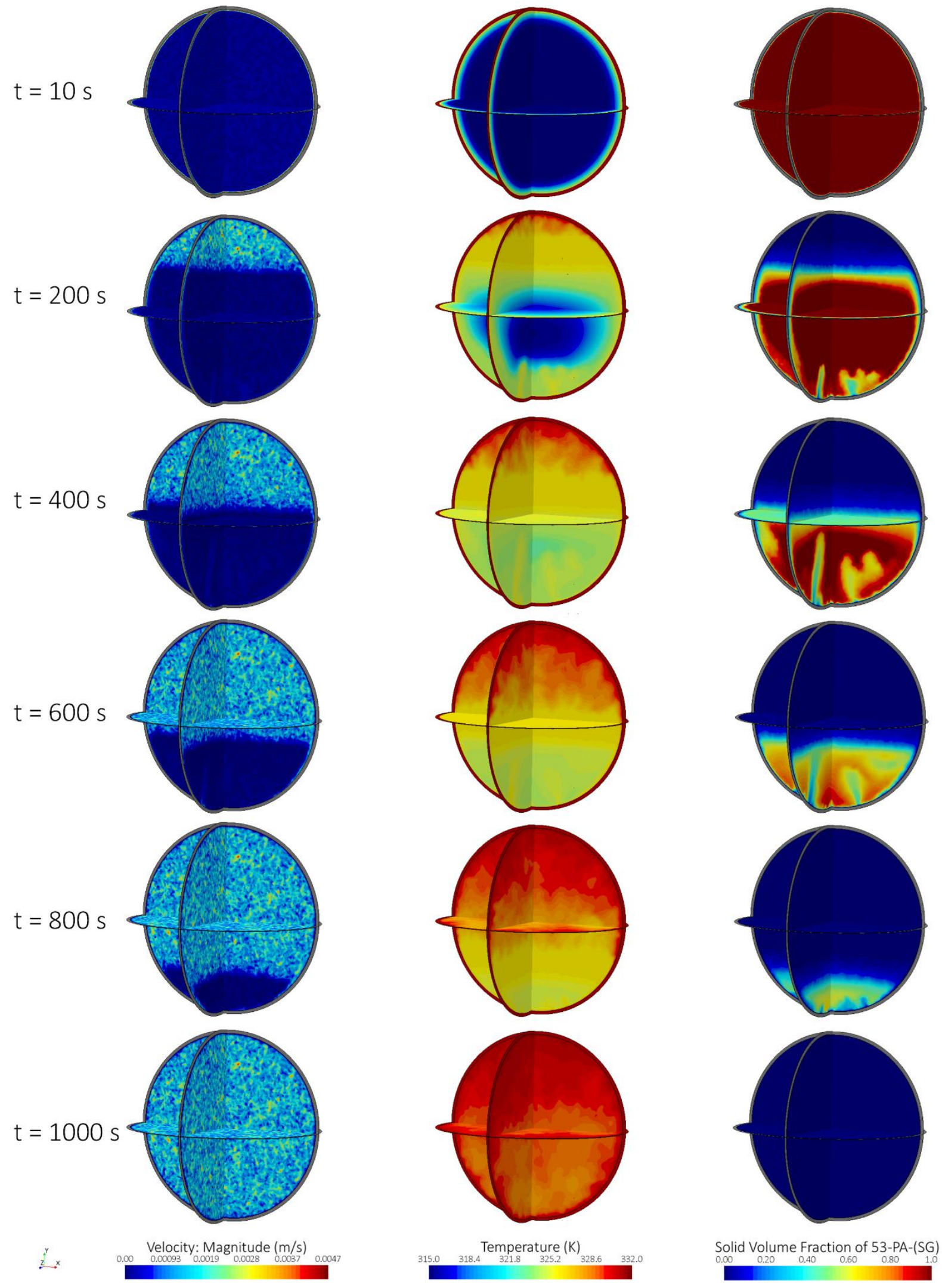

Solid Volume Fraction of 53-PA-(SG)

Fig. 9. Visualization of the melting process every $200 \mathrm{~s}$ of the simulated time t, showing the solid volume fraction $\varphi(-)$, the velocity $\mathbf{u}(\mathrm{m} / \mathrm{s})$ and the temperature $\mathrm{T}(\mathrm{K})$. 
Data in the experiment was collected at one point in the middle at a rate of $1 \mathrm{~s}$. The monitored data in the experiment represents a comparable point. Functions have been created for both, the experiment and the simulation, to compensate small deviations better. These can be seen as dotted and dashed lines of the same color.

Especially before the melting, both graphs show a good agreement with one another. During the melting, at temperatures of $\mathrm{T}=324 \mathrm{~K}$ up to $\mathrm{T}=326 \mathrm{~K}$, there are deviations between the experimental data and the simulation. The non-conformance here is less than $0.5 \mathrm{~K}$. After the melting process is finished, at $\mathrm{T}>326 \mathrm{~K}$, the temperature in the simulation rises about $70 \mathrm{~s}$ earlier than the PCM's temperature in the experiment. The course of the simulation curve corresponds with the course of the experiment's curve. This indicates that the measuring point in the simulation was chosen with a slightly too high y-axis value. Small deviations throughout the measurement could be caused by inaccuracy of the temperature sensor in the test stand. The overall agreement of experiment and simulation is close and therefore, the simulation model can be further used for validations of the numerical model to describe solidification processes

The overall melting process is demonstrated in Fig. 9. Velocity magnitude $\mathbf{u}$, temperature $\mathrm{T}$ and solid volume fraction are displayed every $200 \mathrm{~s}$ throughout the melting process. When looking at the temperature progress in the middle, the steady temperature in the solid fraction of the PCM can be monitored. As the first parts of the PCM are already melted at $\mathrm{t}=200 \mathrm{~s}$ there is still part of the PCM not heated up. This is caused by the poor thermal conductivity of the PCM. Areas with higher temperatures also have a $\mathrm{SVF}=0$ and small velocities $\mathbf{u}<0.0047 \mathrm{~m} / \mathrm{s}$. These velocities are caused by thermal convection and gravity movements of the solid phase. As it can be seen in the right column, where the SVF is depicted, the melting front stays relatively small until $t=600 \mathrm{~s}$, leaving high $\varphi$ values (dark red) until far into the melting process. Starting at $t=200 \mathrm{~s}$ irregularities along the melting front develop and curve into the material. These could be caused by irregularities in the used numerical grid and need further investigation, as they are not observed in the experiment.

Overall the downwards movement of the PCM can clearly be seen. This is a result of gravity, the lower viscosity and density of the liquid phase compared to the solid phase. On the other side within the solid phase the viscosity models with a viscous resistance of $10^{6} \mathrm{~kg} / \mathrm{m}^{3} \mathrm{~s}$ simulate the solid material of the PCM accurately. As Kasibhatla et al. [4] find high viscosities of $10^{5}$ better than viscosities of $10^{3}$ in their simulation model, this approach aimed for a high viscous resistance. The viscous resistance of $10^{6} \mathrm{~kg} / \mathrm{m}^{3} \mathrm{~s}$ has an excellent agreement for SVF values of $\varphi>0.27$.

\section{CONCLUSION AND OUTLOOK}

The article focuses on a validation of a numerical model for solid-liquid phase change problems with variable material data. The here proposed model for melting processes of PCMs shows good agreement to the experimental data. The mushy zone model in combination with variable material data is able to reproduce accurate melting processes numerically. Further investigation will consider different melting ranges and solidification processes. The here proposed numerical model can be the foundation to find thermal conductivity formulations to simplify CFD models further. It is the aim of further studies to reduce simulation time, in order to be able to simulate hybrid thermal storages with low numerical effort. With such models thermal storages can be optimized quicker, more accurate and with far less experimental effort.

As thermal storages with higher capacities and efficiencies are strongly needed now and in the future, it is essential to be able to develop and optimize such systems with lower effort as today. Deeper insight on thermal processes of PCMs and thermal storages can improve the development of combined LHS and SHS and therefore feed the strong demand of improved thermal energy storages.

\section{ACKNOWLEDGMENT}

The present work was done within the framework program InnoSÜD. The authors acknowledge the financial support of the project InnoSÜD by the Federal Ministry of Education and Research of Germany (BMBF) and the State government in the framework of the Innovative Hochschule.

\section{REFERENCES}

[1] T. Fleiter et al., "Profile of heating and cooling demand in 2015," 2017.

[2] H. Mehling and L. F. Cabeza, Heat and cold storage with PCM: An up to date introduction into basics and applications ; with 28 tables. Berlin, Heidelberg: Springer, 2008

[3] Z. Liu, Y. Yao, and H. Wu, "Numerical modeling for solid-liquid phase change phenomena in porous media: Shell-and-tube type latent heat thermal energy storage," Applied Energy, vol. 112, pp. 1222-1232, 2013

[4] R. R. Kasibhatla, A. König-Haagen, F. Rösler, and D. Brüggemann, "Numerical modelling of melting and settling of an encapsulated PCM using variable viscosity," Heat Mass Transfer, vol. 53, no. 5.

[5] J. Vogel and A. Thess, "Validation of a numerical model with a benchmark experiment for melting governed by natural convection in latent thermal energy storage," Applied Thermal Engineering, vol. 148, pp. 147-159, 2019.

[6] N. R. Vyshak and G. Jilani, "Numerical analysis of latent heat thermal energy storage system," Energy Conversion and Management, vol. 48, no. 7, pp. 2161-2168, 2007.

[7] M. Dzikevics, A. Ansone, and D. Blumberga, "Modelling of Phase Change in Spheres for Applications in Solar Thermal Heat Storage Systems," Energy Procedia, vol. 95, pp. 112-118, 2016.

[8] M. A. Izquierdo-Barrientos, C. Sobrino, and J. A. AlmendrosIbáñez, "Modeling and experiments of energy storage in a packed bed with PCM," International Journal of Multiphase Flow, vol. 86, pp. 1-9, 2016.

[9] J. Yang, L. Yang, C. Xu, and X. Du, "Experimental study on enhancement of thermal energy storage with phase-change material," Applied Energy, vol. 169, pp. 164-176, 2016.

[10] Z. Wang, H. Zhang, B. Dou, H. Huang, W. Wu, and Z. Wang, "Experimental and numerical research of thermal stratification with a novel inlet in a dynamic hot water storage tank," Renewable Energy, vol. 111, pp. 353-371, 2017.

[11] V. Pabst, G. Mengedoht, and P. Renze, "A Comparison of Stratified Heat Storage With and Without Modular PCM Storage Through Simulation," in Proceedings of EuroSun 2018, Rapperswil, CH, Sep. 2018 - Sep. 2018

[12] N. Zhang, Y. Yuan, X. Cao, Y. Du, Z. Zhang, and Y. Gui, "Latent Heat Thermal Energy Storage Systems with Solid-Liquid Phase Change Materials: A Review," Adv. Eng. Mater., vol. 20, no. 6, p. 1700753, 2018

[13] C.W. Hirt and B.D. Nichols, "Volume of fluid (VOF) method for the dynamics of free boundaries," Journal of Computational Physics, vol. 39, no. 1, pp. 201-225, 1981 
[14] D.-L. Sun, J.-L. Xu, and L. Wang, "Development of a vapor-liquid phase change model for volume-of-fluid method in FLUENT," International Communications in Heat and Mass Transfer, vol. 39, no. 8, pp. 1101-1106, 2012

[15] J. D. Anderson, Computational fluid dynamics: The basics with applications, 8th ed. New York: McGraw-Hill, 2001.

[16] Siemens PLM Software, STAR-CCM+, 2017.

[17] P. C. Carman, "Fluid Flow Through Granular Beds," in Transactions of the Institution of Chemical Engineers, pp. 150-166.

[18] A. B. Metzner, "Rheology of Suspensions in Polymeric Liquids," Journal of Rheology, vol. 29, no. 6, pp. 739-775, 1985

[19] S. Chang and D. M. Stefanescu, "A model for macrosegregation and its application to Al-Cu castings," MMTA, vol. 27, no. 9, pp. 27082721, 1996 